\title{
Die Welt von Gestern und ihre Widersacher: (Dis)kontinuitäten in der österreichischen Nachkriegsliteratur
}

\section{The World of Yesterday and Its Adversaries: (Dis)continuities in Post-war Austrian Literature}

Juliane Werner

\begin{abstract}
The article The World of Yesterday and Its Adversaries: (Dis)continuities in Post-war Austrian Literature provides an outline of the complex interplay of ruptures and continuities in Austrian literature since 1945 and relates them to the historical context. After a phase of catching up on international trends, the Austrian readership largely leaned towards the literary past (of the Austro-Hungarian and the interwar period), encouraged by the cultural policies of the Allies who - operating on the basis of the victim theory - endorsed a cultural heritage that would strengthen the overall sense of community among the Austrian population. After 1948, the lack of reorganization of the literary field became evident in the dominance of catholic conservative agents who upheld their pre-war cultural ideals, and the reintegration of (lesser) Nazi offenders into the literary scene, all of this to the disadvantage of unknown, progressive, and avantgarde writers, whose publishing opportunities were scarce. The article explores how the new generation's various ways of confronting the past represent significant breaks in this continuity, while becoming a major literary trend in their own right.
\end{abstract}

\section{Keywords}

post-1945 Austrian fiction; allied-occupied Austria; victim theory; cultural identity; (dis)continuities; literary commitment 


\title{
1. Alliierte Kulturpolitik und Kontinuität
}

„Nach dem Krieg“ - dies ist die Zeitrechnung“" , setzt das Ich in Ingeborg Bachmanns Erzählung Unter Mördern und Irren (1961) an und sät mit der Betonung des Nachher sogleich - wie sich schnell herausstellt berechtigte - Zweifel am Vorbeisein des Vorher. Auf fehlende innere Brüche und das Fortbestehen faschistischen Gedankenguts nach Ende des Nationalsozialismus hinzuweisen, sieht Bachmann als eine genuine Leistung der Literatur, wie sie in der Vorrede zu Der Fall Franza verdeutlicht:

\begin{abstract}
„Die Massaker sind zwar vorbei, die Mörder noch unter uns, oft beschworen und manchmal festgestellt, nicht alle, aber einige, in Prozessen abgeurteilt. Die Existenz dieser Mörder ist uns allen bewußt gemacht worden, nicht durch mehr oder minder verschämte Berichterstattung, sondern eben auch durch die Literatur.“"
\end{abstract}

Gemeint ist damit weniger die etablierte Literatur der Nachkriegsjahre als jene einer überwiegend jungen Minderheit, die sich gegen das rasch um sich greifende Vergessen des Gestern richtet und deren Art des Schreibens der von der alliierten Kulturpolitik geförderten allgemeinen Rückkehr zur Vorkriegstradition zuwiderläuft. Durch die verschiedenen Kontinuitätserscheinungen in der Nachkriegskultur und -mentalität macht sich 1945 als „Datum einer neuen Zeitrechnung“ vor allem im Alltag bemerkbar, so Wendelin Schmidt-Dengler, ist jedoch „nicht unbedingt in jeder Hinsicht das Jahr Null“3.

Der politische Neuanfang vollzieht sich auf der Basis des österreichischen Opferstatus, der, festgelegt bei der Moskauer Außenministerkonferenz der britischen, sowjetischen und amerikanischen Regierung, das Land zum ersten freien Land, das Adolf Hitler zum Opfer gefallen ist, macht. ${ }^{4}$ Mit der Schuld schwindet auch die Notwendigkeit von Sühne, die die Mitschuldklausel in der Declaration on Austria vom 30. Oktober 1943 durchaus einfordert: „Austria is reminded, however, that she has a responsibility which she cannot

1 Bachmann, Ingeborg (2010): Unter Mördern und Irren. In: Bachmann, Ingeborg: Sämtliche Erzählungen (8. Aufl.). München: Piper, S. 159-186 (hier S. 159). [Zuerst in: Das dreißigste Jahr, 1961]. Dieser Beitrag, bei dem es sich um die Ausarbeitung eines 2019 in Brünn gehaltenen Vortrags handelt, stützt sich auf Erkenntnisse aus: Werner, Juliane (2021): Existentialismus in Österreich. Kultureller Transfer und literarische Resonanz. Berlin - Boston: De Gruyter. [= Studien und Texte zur Sozialgeschichte der Literatur; Bd. 153].

2 Bachmann, Ingeborg (1986): Der Fall Franza. Requiem für Fanny Goldmann (5. Aufl.). München: dtv, S. 9f. Mit dem nicht näher definierten „unter uns“ ließen sich auch die SchriftstellerInnen selbst meinen, die sich laut Wendelin Schmidt-Dengler nach 1945 zwar meist „entschieden von den Nazis distanzierten, aber, und das möchte ich besonders hervorheben, nicht von dem, was der Nazi in ihnen war: Der Umgang mit der eigenen Haltung wurde doch eher marginalisiert." Schmidt-Dengler, Wendelin (1996): Bruchlinien. Vorlesungen zur österreichischen Literatur 1945 bis 1990 (2. Aufl.). Salzburg: Residenz, S. 22. Auf Schmidt-Denglers Bruchlinien sei hiermit für einen detaillierteren Überblick über die Kontinuitäten und Zäsuren der österreichischen Nachkriegsliteratur verwiesen, ebenso auf: Zeyringer, Klaus (2008): Österreichische Literatur seit 1945. Überblicke, Einschnitte, Wegmarken (ergänzte und aktualisierte Neuausgabe). Innsbruck: Studienverlag.

3 Schmidt-Dengler [Anm. 2], S. 16.

4 United States Department of State (1963): Declaration on Austria. In: Franklin, William M. - Perkins, E. R. (hrsg.): Foreign Relations of the United States: Diplomatic Papers, 1943. General, Vol. 1. Washington: Government Printing Office, S. 761 (Annex 6): „the first free country to fall victim to Hitlerite aggression“. 
evade for participation in the war on the side of Hitlerite Germany, and that in the final settlement account will inevitably be taken of her own contribution to her liberation. ${ }^{\text {}} 5$ Dieser Part, sowie sonstige Hinweise darauf, dass die Einverleibung Österreichs in das nationalsozialistische Deutsche Reich im März 1938 von der österreichischen Politik und Bevölkerung in nennenswertem Maße mitgetragen wurde, finden wenig Berücksichtigung; in der unmittelbaren Nachkriegszeit beruft man sich parteiübergreifend auf die neue Selbstdefinition als „erstes von der Welt im Stiche gelassenes Opfer“6, wie es in dem von der Regierung in Auftrag gegebenen Legitimationsband Gerechtigkeit für Österreich! Rot-Weiss-Rot-Buch von 1946 heißt. Die Opferthese wird zum identitätsstiftenden Gründungsmythos der Zweiten Republik, an deren Beginn - wie bei allen „radikale[n] Umbruchsituationen“ und „Orientierungskrisen“7, in denen tradierte Werte ihre Gültigkeit verlieren - ein Bedürfnis nach Mythen besteht, die die Leerstellen befüllen, wie Stephanie Wodianka in Mythos und Tabula rasa (2018) unterstreicht. Am Beispiel des Opfernarrativs veranschaulicht sich, wie Mythen vereindeutigen, „konkurrierende Bedeutungen“ ausblenden und somit „wahr und falsch zugleich“ sind: wahr, indem sie „soziale und politische Wirklichkeiten begründen“ und „der Gemeinschaft eine Zukunft weisen“, falsch, indem sie „fälschen oder etwas verschweigen"8 - Auslassungen, die die Literatur bald aufgreifen wird.

Die Alliierten, die in ihren jeweiligen Besatzungszonen im Sinne der politischen Vorgabe auch die Entnazifizierung „mit einem gewissen Wohlwollen“9 betreiben, haben Gründe für ihr schonendes Vorgehen. Angrenzend an mehrere kommunistische Staaten hat Österreich eine strategisch wichtige Position in dem sich ankündigenden Kalten Krieg, weshalb es seine Funktionalität und Integrität schnellstmöglich wiederherzustellen gilt. Um das Land dauerhaft von jedem Anschlusswunsch an Deutschland zu befreien, werden in den ersten Besatzungsjahren nahezu alle Verbindungen gekappt. Die bloBe Erwähnung Deutschlands war „unpopulär“10, so Hans Weigel in seinem provokanten Artikel Das verhängte Fenster (1946) über die Trennung vom westdeutschen Buchmarkt, welche ihm aufgrund der Gleichsprachigkeit gerade im Bereich der seinerzeit oft kaum

$5 \quad$ United States Department of State [Anm. 4], S. 761.

6 o. V. (1946): Gerechtigkeit für Österreich! Rot-Weiss-Rot-Buch. Darstellungen, Dokumente und Nachweise zur Vorgeschichte und Geschichte der Okkupation Österreichs (nach amtlichen Quellen). Wien: Verlag der österreichischen Staatsdruckerei, S. 5 (Hervorhebung im Original).

7 Wodianka, Stephanie (2018): Mythos und Tabula rasa: Poetik des Erzählens, Erinnerung und Gedächtnis, Fläche und Raum. In: Bizeul, Yves - Wodianka, Stephanie (hrsg.): Mythos und Tabula rasa. Narrationen und Denkformen der totalen Auslöschung und des absoluten Neuanfangs. Bielefeld: transcript, S. 25-39 (hier S. 26).

8 Wodianka [Anm. 7], S. 25-26.

9 Klöckler, Jürgen (2004): Ici L'Autriche - Pays Ami! Frankreich und die Entnazifizierung in Österreich 1945/46. In: Schuster, Walter - Weber, Wolfgang (hrsg.): Entnazifizierung im regionalen Vergleich. Linz: Archiv der Stadt Linz, S. 455-472 (hier S. 470). Cf. zu den für die Literatur relevanten Maßnahmen die Aufsätze von Gerhard Renner (Entnazifizierung der Literatur, S. 203-229), und Murray G. Hall (Entnazifizierung in Buchhandel und Verlagen, S. 230-253) in: Meissl, Sebastian - Mulley, Klaus-Dieter - Rathkolb, Oliver (hrsg.) (1986): Verdrängte Schuld, verfehlte Sühne. Entnazifizierung in Österreich 1945-1955 [= Symposion des Instituts für Wissenschaft und Kunst, Wien, März 1985]. Wien: Verlag für Geschichte und Politik.

10 Weigel, Hans (1946): Das verhängte Fenster. In: Plan 1, Nr. 5, S. 397-399 (hier S. 397). 
zu bezahlenden Übersetzungsrechte unökonomisch erscheint. Erst 1948 wird der Handel mit Deutschland, wo sich in den Folgejahrzehnten viele österreichische SchriftstellerInnen verlegen lassen, langsam wieder aufgenommen. „[N]ie war ein Land exotischer als dieses Deutschland“, erinnert sich Ingeborg Bachmann noch vier Jahre später in einem Entwurf über die Gruppe 47:

„Im Jahr 1952 wußte man in Österreich so gut wie nichts über neue deutsche Schriftsteller. Hin und wieder fuhr jemand, der Paß und Visum hatte, nach Deutschland, das ferner schien als jedes andre Land, einige wenige ,Pioniere', die dann erzählten, es wäre zuviel gesagt, daß man [aus] jenen Erzählungen klug hätte werden können“..11

Bachmanns Zitat bestätigt ex post das Gelingen der auf Isolation setzenden Besatzungsmaßnahmen, die auf den Aufbau eines Staats- und Selbstbewusstseins Österreichs zielen, im Eilverfahren, gegeben, dass derartige ,[k]ollektive Mentalitäts- und Traditionsbildungen“ üblicherweise „Prozesse von langer Dauer"12 sind. Im Zuge des nation building geht die Konstruktion eines autonomen Österreich Hand in Hand mit der Konstruktion einer autonomen österreichischen Literatur. Die große eigene Vergangenheit, die sich vor allem in der Kultur der Habsburgerzeit materialisiert, bietet sich angesichts der kulturell, soziopolitisch und wirtschaftlich instabilen Gegenwart als bester Referenzpunkt für die Stärkung eines Gemeinschaftsgefühls an. Das Anknüpfen an die glanzvolle Tradition früherer Tage erfolgt unter nicht weiter kommentierter Ausklammerung der Zeit des Nationalsozialismus, die etwa Émile Béthouart, Hochkommissar der französischen Alliierten, als siebenjährigen Schlummerzustand („état de léthargie“13) versteht, aus dem das Land nun behutsam geweckt werden müsse. Das Zurückblicken wird vor allem von VorkriegsschriftstellerInnen befürwortet, ausdrücklich etwa von Alexander Lernet-Holenia, der 1945 seine Haltung als Gruß des Dichters in der Monatsschrift Der Turm verewigt:

„Um es vollkommen klar zu sagen: wir haben es nicht nötig, mit der Zukunft zu kokettieren und nebulose Projekte zu machen, wir sind, im besten und wertvollsten Verstande, unsere Vergangenheit, wir haben uns nur zu besinnen, daß wir unsere Vergangenheit sind - und sie wird unsere Zukunft werden. “14

11 Bachmann, Ingeborg (1978): [Gruppe 47] Entwurf. In: Bachmann, Ingeborg: Werke, Bd. 4: Essays, Reden, Vermischte Schriften, Anhang. Hrsg. von Christine Koschel, Inge von Weidenbaum und Clemens Münster. München - Zürich: Piper, S. 323-330 (hier S. 325, 323). Cf. zur Öffnung des Buchmarktes: McVeigh, Joseph G. (1996): Lifting the Paper Curtain: The Opening of Austrian Literary Culture to Germany after 1945. In: German Studies Review 19, Nr. 3, S. 479-499. Cf. weiters: Lunzer, Heinz (1984): Der literarische Markt 1945-1955. In: Aspetsberger, Friedbert - Frei, Norbert - Lengauer, Hubert (hrsg.): Literatur der Nachkriegszeit und der fünfziger Jahre in Österreich. Wien: ÖBV. [= Schriften des Institutes für Österreichkunde 44/45], S. 24-45.

12 Rosenberg, Rainer (2003): Verhandlungen des Literaturbegriffs. Studien zu Geschichte und Theorie der Literaturwissenschaft. Berlin: Akademie Verlag, S. 178.

13 Haut Commissariat de la République Française en Autriche; Division Information, Centre de Documentation (1948): Deux ans et demi de présence française en Autriche. Notes documentaires et études $\mathrm{N}^{\circ}$ 870; Série européenne - CXIV, S. I. [Übers. d. Verf.]

14 Lernet-Holenia, Alexander (1945): Gruß des Dichters. In: Der Turm 1, Nr. 4/5, S. 109. (Hervorhebung im Original). McVeigh betont, dass ,[t] $]$ he act of reconstituting Austria's literary past in order to utilize traditions 
Auf welchen Teil der literarischen Vergangenheit man sich besinnen soll, wird verschiedentlich beantwortet, für den konservativen (mit folgendem Vorschlag aber durchaus zur fortschrittlicheren Fraktion gehörenden ${ }^{15}$ ) Literaturkritiker und Schriftsteller Herbert Eisenreich zählt die bislang nicht ausreichend zugängliche beziehungsweise gewürdigte „große Generation vorher“ jedenfalls noch dazu; er denkt

„an den geheimnisvollen Humor Kafkas und an die intellektuelle Skepsis Musils, an die von der Sprache erhellte Untergangsstimmung Trakls und an die Sprach- und Sachkritik von Karl Kraus, an die Vivisektion der Gesellschaft durch Broch, an den Weltblick Hofmannsthals. "16

Diese Auswahl zum literarischen Leitbild zu erheben, hätte durch ihren habsburgisch-übernationalen Kern zudem den Vorteil, die von den Alliierten eingeführten Werke aus den USA, England und Frankreich ablösen zu können, die seit Beginn der Besatzungszeit die geistigen Vakua füllen.

\section{Vom Nachholbedarf zum neuen Kanon}

Zu den nach 1945 vorherrschenden materiellen Nöten kommt nach siebenjähriger Abschirmung vom internationalen Kulturgeschehen ein erheblicher immaterieller Mangel, wie der Journalist Friedrich Heer, ein Zeitzeuge, berichtet:

„Begierig wurde versucht, einen Nachholbedarf zu stillen. Also: die Dichter, Schriftsteller, Denker Frankreichs, Englands, Amerikas hereinzuholen, in ihren Werken zumindest, als Geburtshelfer für ,die Neue Zeit'،"17

Die Alliierten kommen diesem Bedürfnis so gut wie möglich nach mit der Förderung, Publikation und sonstigen Vermittlung (speziell Besprechungen in der Besatzungspresse) beliebter und idealerweise wenig polarisierender ausländischer Werke, wobei die weitgehend unbekannte angloamerikanische Moderne (darunter Faulkner, Greene, Hemingway, Steinbeck) und die jüngeren französischen Strömungen Renouveau Catholique und (der wegen seiner Drastik mit Vorsicht vorgestellte) Existentialismus

and tendencies from the prewar years to the advantage of present cultural and political exigencies", als erklärtes Ziel des Unterrichtsministeriums unverzüglich in der schulischen Literaturgeschichtsschreibung umgesetzt wird. McVeigh, Joseph (1988): „Das bin nur ich. Wenn ich es bin“: Politics and Literature in Austria after 1945. In: The German Quarterly 61, Nr. 1, S. 5-21 (hier 9).

15 Auch 1963 rät noch der experimentell dichtende Walter Buchebner der Jugend, sich literarisch in den „übernationalen Raum, den der alte Völkerstaat der Donaumonarchie [...] hinterlassen hat“, zu begeben. Buchebner, Walter (1963): Lektüre-Ratschläge für die junge österreichische Generation. In: Wort in der Zeit 9, Nr. 7, S. 41-48 (hier S. 48).

16 Eisenreich, Herbert (1954): Worin besteht der Unterschied? Ein Vergleich zwischen der jungen Literatur Österreichs und Deutschlands. In: Forum 1, Nr. 7/8, S. 34-36 (hier S. 35).

17 Heer, Friedrich (1985): Nach 1945. In: Jung, Jochen (hrsg.): Vom Reich zu Österreich. Kriegsende und Nachkriegszeit in Österreich erinnert von Augen- und Ohrenzeugen. München: dtv, S. 150-161 (hier S. 158). 
(u. a. Bernanos, Camus, Péguy, Mauriac, Sartre) auf besonderes Interesse stoßen. Die sich damit ergebende Internationalisierung des literarischen Feldes wird mitgetragen von zahlreichen neu gegründeten, meist kurzlebigen Zeitschriften - etwa der dem Innsbrucker Institut Français zugeordneten Wort und Tat (1946-1948), aber auch unabhängigen Blättern wie Otto Basils Plan (1945-1948) - die sich neben ersten kurzen Veröffentlichungen der jungen Generation vor allem der Vermittlung internationaler Literatur widmen.

Dass die genannten Periodika das Jahr 1948 nicht überstehen, ist kein Zufall. Als erstes ,Normaljahr“ nach dem Krieg markiert es einen deutlichen Umbruch: Der Nachholbedarf scheint einigermaßen gedeckt, Versäumtes aufgeholt, das Interesse des Publikums schwindet und mit ihm auch die Aufnahmemöglichkeiten. Viele Zeitschriften geraten überdies in Schwierigkeiten durch die vermehrte Konkurrenz von Zeitungen seit der Überwindung der ersten Papierknappheit oder fallen der Währungsreform Ende 1947 zum Opfer, die auch die Verlage in eine Krise stürzt, da die zur wirtschaftlichen Stabilisierung nötige Reduktion der Geldmenge deren Produkte um das Zwei- bis Dreifache verteuert. Dieser Umstand und die Wiederverfügbarkeit anderer Waren lassen ebenfalls die Nachfrage am anfänglich durch den allgemeinen Konsumgüter-Mangel äußerst begehrten Buch sinken. Auch die Umstrukturierungen im Literaturbetrieb, der zunehmende Rückzug der Alliierten, der Wiedereintritt konservativer Vorkriegs- und KriegsakteurInnen und die Verdrängung der im antikommunistischen Klima immer unliebsamer werdenden (pro-)kommunistischen Kräfte, etwa aus dem PEN-Umfeld, sorgt für eine Abwendung von neu(er)en nationalen und internationalen Literaturerscheinungen. ${ }^{18}$ Das Erheben des Erbes der ersten Jahrhunderthälfte (vor allem Kafka, Musil, Trakl, Broch) zum Neuen gehört zu den Idiosynkrasien des österreichischen Neuanfangs, die in Hans Weigels Augen höchstens oberflächlich besehen paradox erscheinen:

„Die große optische Täuschung jeder Mitwelt besteht darin, Lebende unter allen Umständen für lebendig, Gestorbene für tot und Gleichalte für gleich alt zu halten. Noch ist das neunzehnte Jahrhundert unter uns, in Sechzig- und Siebzigjährigen, das zwanzigste aber steht vor uns in Trakl, Kafka und anderen großen Lebendigen." ${ }^{19}$

Die aufregendste Entdeckung dieser Jahre ist Franz Kafka. Es gibt, wie Friedrich Torberg hervorhebt, bis zu dessen 30. Todestag 1954 „kaum einen zweiten Autor der leidenschaftlicher diskutiert und vielfältiger interpretiert wird“20, wobei die diversen Verein-

18 Cf. Maurer, Stefan - Neumann-Rieser, Doris - Stocker, Günther (hrsg.) (2017): Diskurse des Kalten Krieges. Eine andere österreichische Nachkriegsliteratur. Wien - Köln - Weimar: Böhlau. [= Literaturgeschichte in Studien und Quellen 29]. Cf. für die sich wandelnde Rolle des PEN-Clubs in den Nachkriegsjahren die Aufsätze von Klaus Amann (Der österreichische PEN-Club in den Jahren 1923-1955, S. 481-532) und Ingrid Schramm (Der Wiener PEN-Club vom Beginn des Kalten Krieges bis zur Ostöffnung [1947-1990], S. 533-549), in: Bores, Dorothée - Hanuschek, Sven (hrsg.) (2014): Handbuch PEN. Geschichte und Gegenwart der deutschsprachigen Zentren. Berlin - Boston: De Gruyter.

19 Weigel, Hans (1953): Die gefundene Generation: In: Kontinente 7, Nr. 1, S. 13-14 (hier S. 13).

20 [Torberg, Friedrich] tbg. (1954): Zur 30. Wiederkehr des Todestages von Franz Kafka. In: Forum 1, Nr. 6, S. 19. 
nahmungen an Adornos Bezeichnung Kafkas als „Auskunftsbüro“21 für alle Belange denken lassen. Stolz auf „die Ideen des Österreichers Kafka, der ein dichtender Philosoph war“, ist man etwa bei dem von der amerikanischen Besatzungsmacht gegründeten Wiener Kurier, der den Prager Autor zu einem „Hauptvertreter der neuen Existenzphilosophie“ macht, die man „in der geistigen Welt Frankreichs, Englands und Amerikas“22 debattiert. In der österreichischen Rezeption werden Kafkas Themen - das Verschwinden verlässlicher Kausalitäten, die Fremdartigkeit des Lebens, Verurteilungen ohne Anklagen, Bestrafungen ohne Schuld - mit dem Existentialismus und dem (hier noch neuen) Surrealismus amalgamiert und zur häufig nachgeahmten Folie für NachwuchsschriftstellerInnen, die Anfang der Fünfzigerjahre „ihre Skepsis, ihre Verlorenheit und Weltangst à la Kafka“23 ausdrücken. Die Kafka-Imitationen, deren bedenkliches Ausmaß Hans Heinz Hahnl Sorge bereitet, denn das Vorbild ist „kaum abzuwerfen, unmöglich zu überwinden“24, bleiben ohne nennenswerten Erfolg. Wird Kafka - wie auch Musil und Broch - „für die meisten nach 1945 Schreibenden verbindlich“25, wie Schmidt-Dengler anmerkt, so ist damit die junge Generation gemeint, die aufgrund des maßgebenden konservativen Literaturideals vom Durchbruch mehrheitlich noch weit entfernt ist.

\section{Stimmen der Vergangenheit und der Gegenwart}

Die „Restauration katholischen Geisteslebens“26, als Hauptpfeiler der Nachkriegskontinuität, verwirklicht sich mithilfe von harmlose(re)n RepräsentantInnen des Vorkriegsliteraturbetriebs, die ihre Tätigkeit fortzusetzen beabsichtigten. Die teilweise Rekonstruktion der ständestaatlichen Literatur und Literaturpolitik wird ab 1948/49 ergänzt durch die vorübergehend aus dem literarischen und verlegerischen Verkehr Gezogenen: Von der 1946 vom Bundesministerium für Unterricht ausgegebenen Liste der gesperrten Autoren und Bücher, auf der sich Namen wie Gertrud Fussenegger, Friedrich Schreyvogl,

21 Adorno, Theodor W. (1996): Aufzeichnungen zu Kafka. In: Adorno, Theodor W.: Kulturkritik und Gesellschaft I [= Gesammelte Schriften 10.1] (2. Aufl.). Hrsg. von Rolf Tiedemann. Frankfurt am Main: Suhrkamp, S. 254-287 (hier S. 254).

$22-$ n (1946): Zwei Bücher aus Österreich. Zur weltanschaulichen Situation unserer Zeit. In: Wiener Kurier, 28. 3. 1946.

23 Hahnl, Hans Heinz (1951): Zur Situation der Literatur. In: Stimmen der Gegenwart, S. 20-25 (hier S. 22).

24 Hahnl, Hans Heinz (1985): Von der Diskreditierung der Ideologien. Erwartungshaltungen und literarische Produktion nach 1945. In: Waechter-Böhm, Liesbeth (hrsg.): Wien 1945. davor/danach. Wien: Brandstätter, S. 153-163 (hier S. 159).

25 Schmidt-Dengler, Wendelin (1995): Vorwort. In: Nachbaur, Petra - Scheichl, Sigurd Paul (hrsg.): Literatur über Literatur. Eine österreichische Anthologie. Innsbruck: Haymon, S. 11-24 (hier S. 15).

26 Stadler, Friedrich (1988): Kontinuität und/ oder Bruch? Anmerkungen zur österreichischen Wissenschaftsgeschichte 1938 bis 1955. In: Stadler, Friedrich (hrsg.): Kontinuität und Bruch. 1938 - 1945 - 1955 . Beiträge zur österreichischen Kultur- und Wissenschaftsgeschichte. Wien - München: Jugend und Volk, S. 9-23 (hier S. 11). Cf. auch Dvořak, Johann (1988): Thesen zur soziokulturellen Entwicklung in Österreich 1933 bis 1955. In: Stadler [Anm. 26], S. 27-33. Sowie: Białek, Edward - Żyliński, Leszek (hrsg.) (2006): Die Quarantäne. Deutsche und österreichische Literatur der fünfziger Jahre zwischen Kontinuität und Neubeginn (2. Aufl.). Wrocław - Dresden: Neisse. 
Josef Weinheber, Bruno Brehm und Maria Grengg finden, konnten einige AutorInnen fast bruchlos an ihre Erfolge anknüpfen, weshalb Bachleitner, Eybl und Fischer nicht nur von einer gescheiterten Entnazifizierung, sondern von einer „Renazifizierung ${ }^{“ 27}$ der Literatur sprechen. Hierzu gehören neben der Berücksichtigung in den „meisten ap-

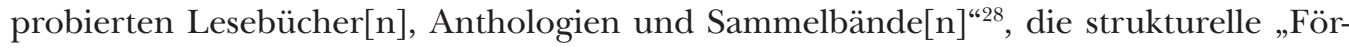
derung von Naziautoren“"29 durch höhere Papierzuteilungen für entsprechende Verlage und durch Literaturpreise, die in den ersten fünfzehn Nachkriegsjahren am öftesten an jene vergeben werden, die „bereits unter dem Austrofaschismus oder unter dem Nati-

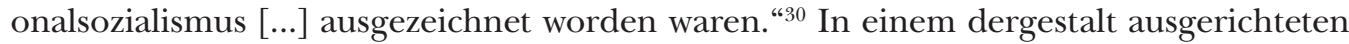
Literaturbetrieb gibt man sich insgesamt „desinteressiert und indolent“"31 der Rückkehr derjenigen gegenüber, die wie Jean Améry oder Manès Sperber vertrieben wurden.

Während die teils belastete ältere AutorInnen-Generation ab circa 1948 die Leerstellen besetzt, die bis dahin durch die internationale Literatur ausgefüllt waren, ist es um die junge still, ihr stehen kaum Publikationsplattformen zur Verfügung. Ihre ersten Schreibversuche werden als unfertig empfunden, ihre Literatur, „die klein daherkommt" ${ }^{\text {"32 }}$, passt nicht in das propagierte Selbstbild als große Kulturnation, die sich besser im vergangenheitszugewandten Monumentalstil Heimito von Doderers wiedererkennt. Doch auch die Taktik jener unbekannten AutorInnen, bei denen die staatliche Förderung konservativen Schreibens eine Selbstzensur in Gang gesetzt hat und deren Texte daher „häufig eine unbewusste Kontinuität“"33 zur Literatur der Ersten Republik aufweisen, geht meist nicht auf und erntet für ihr „müdes ,Fortwursteln““34 den Spott der literarisch vorwärtsgewandten Geister.

Ansonsten dichten die Jungen ins Leere, bestenfalls wird ihnen „von inkompetenten Wichtigtuern des offiziellen Betriebs [...] leutselig auf die Schultern geklopft“35, so Hans

27 Bachleitner, Norbert - Eybl, Franz M. - Fischer, Ernst (2000): Geschichte des Buchhandels in Österreich. Wiesbaden: Harrassowitz. [= Geschichte des Buchhandels; Bd. 6], S. 328.

28 McVeigh, Joseph (1988): Kontinuität und Vergangenheitsbewältigung in der österreichischen Literatur nach 1945. Wien: Braumüller. [= Untersuchungen zur österreichischen Literatur des 20. Jahrhunderts; Bd. 10], S. 78.

29 Schreiber, Hermann (1947): Das Buch. Papierpolitik. In: Österreichisches Tagebuch, 19. 9. 1947. Cf. für eine aufschlussreiche Stellungnahme zur Nachkriegsliteratur im kommunistischen Tagebuch außerdem: Priester, Eva (1946): Die Aufgaben der österreichischen Literatur. In: Österreichisches Tagebuch, 16. 11. 1946.

30 Amann, Klaus (1984): Vorgeschichten. Kontinuitäten in der österreichischen Literatur von den dreißiger zu den fünfziger Jahren. In: Aspetsberger [et al.] [Anm. 11], S. 46-58 (hier S. 47).

31 Schmid, Georg (1984): Die ,Falschen` Fuffziger. Kulturpolitische Tendenzen der fünfziger Jahre. In: Aspetsberger [et al.] [Anm. 11], S. 7-23 (hier S. 19).

32 Scharang, Michael (1986): Die proletarisierte Literatur. In: Scharang, Michael: Die List der Kunst. Essays. Darmstadt - Neuwied: Luchterhand, S. 14-24 (hier S. 15). [Zuerst in: Literatur Konkret 1983/1984, Nr. 8.].

33 Kriegleder, Wynfrid (2014): Die Literatur der fünfziger Jahre in Österreich - ein Überblick. In: treibhaus 10 („Österreich“), S. 29-49 (hier S. 38).

34 Fischer, Ernst (1946): Um ein geistiges Österreich! In: Fischer, Ernst: Das Jahr der Befreiung. Aus Reden und Aufsätzen. Wien: Stern, S. 98-100 (hier S. 98). [Zuerst in: Neues Österreich, 4. 1. 1946].

35 Weigel, Hans (1951): Vorbemerkung. In: Stimmen der Gegenwart, S. 5. Cf. auch Weigel, Hans (1950): Autoren, die uns nicht erreichen. Zur tragischen Situation der jungen österreichischen Literatur. In: Arbeiter-Zeitung, 24. 9. 1950. 
Weigel, ihr größter Förderer. Weigel gibt das Jahrbuch Stimmen der Gegenwart heraus, das zwischen 1951 und 1956 in fünf Bänden „fast alle Autorinnen und Autoren, die in der Folge eine wichtige Rolle in der österreichischen Literatur spielen“"36, lanciert. Mit Gedichten und Erzählungen vertreten sind unter anderem Ingeborg Bachmann, Marlen Haushofer, Paul Celan, Christine Lavant, Michael Guttenbrunner, Gerhard Fritsch, Erich Fried, Christine Busta, Thomas Bernhard, Jeannie Ebner und auch einige der avantgardistischen SchriftstellerInnen, die im Folgejahrzehnt stärker in Erscheinung treten, darunter Andreas Okopenko, H. C. Artmann und Ernst Jandl. Da das Veröffentlichen unbekannter LiteratInnen von verlegerischer Seite in der Wiederaufbauphase der Fünfzigerjahre noch ein zu großes finanzielles Wagnis darstellt, sind ihre Publikationsmöglichkeiten auf Zeitschriften, Anthologien und Jahrbücher beschränkt. Mit desolaten Folgen, wie Weigel 1952 äußert:

„Unbeschreiblich und erschütternd ist ihre materielle Not, ärger noch die Hoffnungslosigkeit und Isoliertheit der von der lebendigen Wirkung in der Öffentlichkeit durch Presse, Bühnen, Verlage, Redaktionen und Sender fast völlig Ausgeschlossenen.“37

Nachdem die Stimmen der Gegenwart-BeiträgerInnen „notorisch geldlos, notorisch hoffnungslos, zukunftslos“38 sind, wie Ingeborg Bachmann aus eigenem Erleben bestätigt, wundert es nicht, dass ihre Texte durch einen recht unumwundenen Pessimismus gekennzeichnet sind, den die sich zuspitzende internationale Bedrohungslage der Epoche noch intensiviert. ${ }^{39}$ Ihre Texte geben Auskunft darüber, dass die offenen Nachkriegsjahre, in denen „die Hoffnung auf eine Umorientierung, auf eine fundamentale Änderung noch möglich schien“40, vorbei sind: Es dominieren eine „erschütternde Ratlosigkeit“, „eine ungeheure Resignation“41, „ein Chaos von Desillusionierung, Zynismus, Fanatismus, Gleichgültigkeit [und] Schwermut“42. Das breite Lesepublikum sucht etwas anderes, Erholung, „Bilder der Einheit, der Ruhe, Geborgenheit und Stabilität“43, und wird fündig in Heimkehr-Romanen, in religiösen Dichtungen und in zeitlos um Natur und Liebe kreisender volkstümlicher Literatur, die ein direktes Anknüpfen an die Vorkriegsidyllen von Karl Heinrich Waggerl, Franz Tumler und Erna Blaas erlaubt.

36 Kriegleder [Anm. 33], S. 39.

37 Weigel, Hans (1953): Vorbemerkung. In: Stimmen der Gegenwart, S. $5-7$ (hier S. 6).

38 Bachmann [Anm. 11], S. 324.

39 Heide Kunzelmann sieht eine große Wichtigkeit in der vom Kalten Krieg verursachten Instabilität für die junge Literatur, ohne die „the productive phenomenon of the fragile equilibrium among the progressive authors would have never realized its full potential at such an early stage." Kunzelmann, Heide (2015): Make or Break? Instability as an Aesthetic Tool in Progressive Austrian Literature from 1945 to 1955. In: Journal of Austrian Studies 48, Nr. 2, S. 89-108 (hier S. 101).

40 Schmidt-Dengler [Anm. 2], S. 26.

41 Basil, Otto (1946): Stimme der Jugend. In: Plan 1, Nr. 4, S. 307-310 (hier S. 307).

42 Hahnl [Anm. 23], S. 21.

43 Müller, Karl (1988): Die Bannung der Unordnung. Zur Kontinuität österreichischer Literatur seit den dreißiger Jahren. In: Stadler [Anm. 26], S. 181-215 (hier S. 187). 
Die jungen SchriftstellerInnen, so Lilly von Sauter, reagieren „aus Protest gegen die übertriebene Gefühlsduselei recht häufig [mit] einer wilden Grausamkeit" ${ }^{44}$ und halten der Sentimentalität Grenztopoi wie Not, Krieg und Folter, Schuld, Kampf, Leid und Tod, Angst und Ohnmacht entgegen. Ilse Aichingers Aussage, die „Erfahrung des nahen Todes“ könne man nicht „wegdenken“45, wird von einer die jüngere Vergangenheit verdrängenden Vergessenskultur bald widerlegt. Dass auch manche der fortschrittlichen KritikerInnen einwenden, dass „aus dem Hinschauen auf Unabänderliches nur eine neue Belastung des schon zur Genüge Bedrückten oder des bisher relativ Unbeschwerten erwachse“46, ist ein Beleg für die These, dass sich die Literatur nach 1945 „nicht über einen Kamm scheren läßt, nicht über den der mangelnden Vergangenheitsbewältigung und der outrierten Restauration, aber auch nicht über den des nachhaltigen Aufbegehrens “ ${ }^{“ 47}$, sondern diese Tendenzen in sich vereint, zum Teil sogar in einzelnen Personen. Interessant in diesem Zusammenhang ist beispielsweise der Wandel Thomas Bernhards, der 1952 zu bedenken gibt, dass Literatur, die von „Krieg und Bombardement, Mord, Flucht und Heimkehr“ handelt, „nicht Zu-, sondern Abneigung“48 bei den LeserInnen auslöst, in den Sechzigerjahren jedoch selbst zu den Schlüsselfiguren der literarischen Kriegskonfrontation gehören wird.

\section{Aufdeckungen und (Anti-)Kontinuität}

Schon bevor die Literatur der Sechzigerjahre schonungslos Verborgenes hervorholt, gibt es neben den Kurztexten in Anthologien auch Romane, die der „Dominanz apolitischer Literaturkonzepte und [der] tendenziellen Abwendung von zeitgeschichtlichen Themen “49 widersprechen, indem sie die österreichische Haltung zur NS-Ideologie vor, im und nach dem Krieg beleuchten, darunter Ilse Aichingers Die größere Hoffnung (1948), Ernst Lothars Die Rückkehr (1949), Milo Dors Tote auf Urlaub (1952), Herbert Zands Letzte Ausfahrt (1953), Fritz Habecks Der Ritt auf dem Tiger (1958) und Reinhard Federmanns Das Himmelreich der Lügner (1959) . Während der „letzte[n] Hochkonjunktur einer Literatur,

44 L. v. S. [Sauter, Lilly von] (1948): Porträt unseres Helden. In: Europäische Rundschau 3, Nr. 17, S. 806-808 (hier S. 807).

45 Aichinger, Ilse (2010): Das Erzählen in dieser Zeit. In Aichinger, Ilse: Der Gefesselte. Erzählungen (19481952). Frankfurt am Main: Fischer, S. 9-11 (hier S. 10f.).

46 Frank, Claudia (1945): Erinnern oder vergessen. In: Plan 1, Nr. 1, S. 133-134 (hier S. 133).

47 Schmidt-Dengler [Anm. 2], S. 32.

48 Th. B. [Bernhard, Thomas] (2015): Bücher warten auf Dich! In: Bernhard, Thomas: Journalistisches. Reden. Interviews [= Werke, Bd. 22/I: Frühe journalistische Arbeiten; Gerichtssaalberichte; Beiträge in Zeitschriften und Sammelbänden; Leserbriefe, Offene Briefe, Telegramme]. Hrsg. von Wolfram Bayer, Martin Huber und Manfred Mittermayer. Berlin: Suhrkamp, S. 88-90 (hier S. 89). [Zuerst in: Demokratisches Volksblatt, 29. 11. 1952.] Cf. in diesem Band auch den Artikel Junge Dichter in Österreich, S. 13-15. [Zuerst in: Demokratisches Volksblatt, 21. 1. 1952.]

49 Stocker, Günther (2010): Der Kalte Krieg in der österreichischen Literatur. Ein Überblick. In: Hansel, Michael - Rohrwasser, Michael (hrsg.): Kalter Krieg in Österreich. Literatur - Kunst - Kultur. Wien: Zsolnay. [= Profile 17/2010], S. 59-80 (hier S. 62f.). 
die sich auf Themen des ,alten Österreich“ konzentrierte“, bilden diese in ihrer Programmatik des Enthüllens teilweise von der littérature engagée Jean-Paul Sartres geprägten AutorInnen „eine kleine Enklave“, die sich, wie Marion Hussong unterstreicht, „dem vorherrschenden Zeitgeist der Apologetik und Verdrängung“" ${ }^{50}$ entgegenstellt.

Unter denen, die diese Entwicklung in den Sechzigerjahren fortsetzen, wie Hans Lebert, Franz Kain, Thomas Bernhard und Gerhard Fritsch, herrscht verbreitet die Annahme, „daß die Chance zum Umdenken, die die Zäsur des Jahres 1945 gebracht hatte, vertan worden war." ${ }^{\circ 1}$ Ihre Prosa weist jene „Vergiftungserscheinungen“ auf, die Erich Fried zufolge das ausbleibende "Auf-sich-Nehmen und Aufarbeiten“52 von Schuld und Mitschuld unweigerlich mit sich bringt. In Hans Leberts Die Wolfshaut (1960) beispielsweise wird eine zurückliegende Hinrichtung von FremdarbeiterInnen zwar verdrängt, doch tragen die Verantwortlichen ihre Gesichter wie „Masken, durch deren Augenschlitze etwas anderes hervorsah, etwas, das gar nichts zu tun hatte mit dem Staatsbürgerblick jener Leute. “53 Der Name des Dorfes, in dem sich das Geschehen ereignet - Schweigen - summiert das Hauptverhalten der in ihm angesiedelten verschworenen Gemeinschaft, bis ein Außenseiter die trügerische Ruhe durchbricht. Gerhard Fritschs Roman Fasching (1967) weist eine ähnliche Ausgangskonstellation auf, insofern es auch hier für die Allgemeinheit „keinen Sinn [hat], in alten Wunden zu stochern“, und ein kritisch beäugter Einzelgänger dadurch in Gefahr gerät, dass er den dringenden Rat „vergeben und vergessen“54 nicht annimmt. Der ,Fremdkörper“ in Thomas Bernhards Frost (1963) kann im dörflichen Weng neben den fragwürdigen EinwohnerInnen noch die Folgen der „hochexplosive[n] Panzerfäuste“ beobachten: „Fetzen von Kindern“, „zerschossene Uniformen auf den Bäumen und aus dem Tümpel herausschauende steife Hände und Füße“55. Die Atmosphäre - die Verrohung der Menschen, die Unmenschlichkeit der Natur - macht den Roman zu einem Vorläufer der Anti-Heimatliteratur, deren VertreterInnen - wie Franz Innerhofer, Gernot Wolfgruber oder Josef Winkler - ab den Siebzigerjahren die narrativen Bestandteile ländlicher Schauplätze ins Negative wenden und sich so gegen die „Klischees der unschuldigen Natur“ ${ }^{46}$ der nach 1945 neuinszenierten Heimat stellen. Die progressive Literatur des Folgejahrzehnts (an ihrer Spitze einige inzwischen Bekannte wie Thomas Bernhard) verlagert ihren Schwerpunkt weiter von der Innerlichkeit zum Direktangriff auf die in den Nationalsozialismus involvierte Elterngeneration, zu der auch Bundespräsident Kurt Waldheim gehört, dessen unaufgeklärte

50 Hussong, Marion (2000): Der Nationalsozialismus im österreichischen Roman 1945-1969. Tübingen: Stauffenburg [= Stauffenburg-Colloquium; Bd. 52], S. 13, 139.

51 Hussong [Anm. 50], S. 140.

52 Fried, Erich (1984): Nachwort zur ersten Auflage. In: Fried, Erich: Ein Soldat und ein Mädchen. Frankfurt am Main: Fischer, o. S. Cf. auch Hussong [Anm. 50], S. 25.

53 Lebert, Hans (1960): Die Wolfshaut. Hamburg: Claassen, S. 29.

54 Fritsch, Gerhard (1995): Fasching; mit einem Nachwort von Robert Menasse. Frankfurt am Main: Suhrkamp, S. 14, 148.

55 Bernhard, Thomas (2018): Frost [= Werke, Bd. 1]. Hrsg. von Martin Huber und Wendelin Schmidt-Dengler. Frankfurt am Main: Suhrkamp, S. 147.

56 Rabenstein-Michel, Ingeborg (2008): Bewältigungsinstrument Anti-Heimatliteratur. In: Germanica 42, S. 1-11 (hier S. 2). https://journals.openedition.org/germanica/525 (9. 1. 2019). 
SA-Mitgliedschaft in die schriftstellerisch vielfach aufgegriffene ,Waldheim-Affäre' mündet. Es gelingt in den Achtzigerjahren, was den verschiedenen durchaus frontalen Angriffen der Sechzigerjahre - Carl Merz' und Helmut Qualtingers Der Herr Karl als Abrechnung mit dem „unpolitischen Österreicher, der durch Bauernschläue, Ideologieskepsis und Kompromißbereitschaft politische Übergriffe erfolgreich überstand “57, Peter Handkes Publikumsbeschimpfung oder Formen von politisch aufgeladener Sprachskepsis und Aktionismus der Wiener Gruppe - noch nicht gelungen war: eine breitflächige öffentliche Debatte anzustoßen. Nachgeholt wird damit auch das Achtundsechziger-Aufbegehren, das in Österreich „weit weniger politisiert und radikal“ ausfällt als in Frankreich oder in Westdeutschland, was seine Ursache laut Gudrun Braunsperger zum einen „in der verzögerten Vergangenheitsbewältigung, zum anderen im noch nicht abgeschlossenen Prozess der österreichischen Identitätsfindung “58 hat.

So ist der Bruch Mitte der Achtzigerjahre, dessen literarisches Echo bis in die Gegenwart zu vernehmen ist, zu einem nicht unwesentlichen Teil Verdienst jener vom fehlenden Bruch 1945 betroffenen, die längste Zeit auf der Schattenseite der literarischen Förderung und Anerkennung stehenden jungen SchriftstellerInnen. Antretend gegen die restaurativen Verhältnisse im Literaturbetrieb und gegen die Dominanz konservativen, religiös getönten und volkstümlichen Schreibens, weist die neue Generation auf diese Weise trotz ihrer vielgestaltigen Ausdrucksformen seit Ende des Krieges eine eigene Kontinuität auf.

\section{Quellen und Literatur}

Adorno, Theodor W. (1996): Aufzeichnungen zu Kafka. In: Adorno, Theodor W.: Kulturkritik und Gesellschaft I [= Gesammelte Schriften 10.1] (2. Aufl.). Hrsg. von Rolf Tiedemann. Frankfurt am Main: Suhrkamp, S. 254-287.

Aichinger, Ilse (2016): Die größere Hoffnung. Frankfurt am Main: Fischer.

Aichinger, Ilse (2010): Das Erzählen in dieser Zeit. In: Aichinger, Ilse: Der Gefesselte. Erzählungen (1948-1952). Frankfurt am Main: Fischer, S. 9-11.

Amann, Klaus (1984): Vorgeschichten. Kontinuitäten in der österreichischen Literatur von den dreißiger zu den fünfziger Jahren. In: Aspetsberger, Friedbert - Frei, Norbert - Lengauer, Hubert (hrsg.): Literatur der Nachkriegszeit und der fünfziger Jahre in Österreich. Wien: ÖBV. [= Schriften des Institutes für Österreichkunde 44/45], S. 46-58.

Bachleitner, Norbert - Eybl, Franz M. - Fischer, Ernst (2000): Geschichte des Buchhandels in Österreich. Wiesbaden: Harrassowitz. [= Geschichte des Buchhandels; Bd. 6].

Bachmann, Ingeborg (2010): Unter Mördern und Irren. In: Bachmann, Ingeborg: Sämtliche Erzählungen (8. Aufl.). München: Piper, S. 159-186. [Zuerst in: Das dreißigste Jahr, 1961].

57 Landa, Jutta (1988): Bürgerliches Schocktheater. Entwicklungen im österreichischen Drama der sechziger und siebziger Jahre. Frankfurt am Main: Athenäum. [= Literatur in der Geschichte. Geschichte in der Literatur 15], S. 37.

58 Braunsperger, Gudrun (2010): Studentenbewegung in Österreich. In: Benedikt, Michael - Knoll, Reinhold Schwediauer, Franz [et al.] (hrsg.): Verdrängter Humanismus - verzögerte Aufklärung; Bd. 6: Auf der Suche nach authentischem Philosophieren. Philosophie in Österreich 1951-2000. Wien: Facultas, S. 860-863 (hier S. 861). 
Bachmann, Ingeborg (1986): Der Fall Franza. Requiem für Fanny Goldmann (5. Aufl.). München: dtv. Bachmann, Ingeborg (1978): [Gruppe 47] Entwurf. In: Bachmann, Ingeborg: Werke, Bd. 4: Essays, Reden, Vermischte Schriften, Anhang. Hrsg. von Christine Koschel, Inge von Weidenbaum und Clemens Münster. München - Zürich: Piper, S. 323-330.

Basil, Otto (1946): Stimme der Jugend. In: Plan 1, Nr. 4, S. 307-310.

[Bernhard, Thomas] Th. B. (2015): Bücher warten auf Dich! In: Bernhard, Thomas: Journalistisches. Reden. Interviews [= Werke, Bd.22/I: Frühe journalistische Arbeiten; Gerichtssaalberichte; Beiträge in Zeitschriften und Sammelbänden; Leserbriefe, Offene Briefe, Telegramme]. Hrsg. von Wolfram Bayer, Martin Huber und Manfred Mittermayer. Berlin: Suhrkamp, S. 88-90. [Zuerst in: Demokratisches Volksblatt, 29. 11. 1952].

[Bernhard, Thomas] Th. B. (2015): Junge Dichter in Österreich. In: Bernhard, Thomas: Journalistisches. Reden. Interviews [= Werke, Bd. 22/I: Frühe journalistische Arbeiten; Gerichtssaalberichte; Beiträge in Zeitschriften und Sammelbänden; Leserbriefe, Offene Briefe, Telegramme]. Hrsg. von Wolfram Bayer, Martin Huber und Manfred Mittermayer. Berlin: Suhrkamp, S. 13-15. [Zuerst in: Demokratisches Volksblatt, 21. 1. 1952].

Bernhard, Thomas (2018): Frost [= Werke, Bd. 1]. Hrsg. von Martin Huber und Wendelin Schmidt-Dengler. Frankfurt am Main: Suhrkamp.

Białek, Edward - Żyliński, Leszek (hrsg.) (2006): Die Quarantäne. Deutsche und österreichische Literatur der fünfziger Jahre zwischen Kontinuität und Neubeginn (2. Aufl.). Wrocław - Dresden: Neisse.

Bores, Dorothée - Hanuschek, Sven (hrsg.) (2014): Handbuch PEN. Geschichte und Gegenwart der deutschsprachigen Zentren. Berlin - Boston: De Gruyter.

Braunsperger, Gudrun (2010): Studentenbewegung in Österreich. In: Benedikt, Michael - Knoll, Reinhold - Schwediauer, Franz [et al.] (hrsg.): Verdrängter Humanismus - verzögerte Aufklärung; Bd. 6: Auf der Suche nach authentischem Philosophieren. Philosophie in Österreich 1951-2000. Wien: Facultas, S. 860-863.

Buchebner, Walter (1963): Lektüre-Ratschläge für die junge österreichische Generation. In: Wort in der Zeit 9, Nr. 7, S. 41-48.

Dor, Milo (2005): Tote auf Urlaub. St. Pölten - Salzburg: Residenz.

Dvořak, Johann (1988): Thesen zur soziokulturellen Entwicklung in Österreich 1933 bis 1955. In: Stadler, Friedrich (hrsg.): Kontinuität und Bruch. 1938 - 1945 - 1955. Beiträge zur österreichischen Kultur- und Wissenschaftsgeschichte. Wien- München: Jugend und Volk, S. 27-33.

Eisenreich, Herbert (1954): Worin besteht der Unterschied? Ein Vergleich zwischen der jungen Literatur Österreichs und Deutschlands. In: Forum 1, Nr. 7/8, S. 34-36.

Federmann, Reinhard (1993): Das Himmelreich der Lügner. Wien: Picus.

Fischer, Ernst (1946): Um ein geistiges Österreich! In: Fischer, Ernst: Das Jahr der Befreiung. Aus Reden und Aufsätzen. Wien: Stern, S. 98-100. [Zuerst in: Neues Österreich, 4. 1. 1946].

Frank, Claudia (1945): Erinnern oder vergessen. In: Plan 1, Nr. 1, S. 133-134.

Fried, Erich (1984): Nachwort zur ersten Auflage. In: Fried, Erich: Ein Soldat und ein Mädchen. Frankfurt am Main: Fischer, o. S.

Fried, Erich (1984): Ein Soldat und ein Mädchen. Frankfurt am Main: Fischer.

Fritsch, Gerhard (1995): Fasching; mit einem Nachwort von Robert Menasse. Frankfurt am Main: Suhrkamp.

Habeck, Fritz (1961): Der Ritt auf dem Tiger (3. Aufl.). Hamburg - Wien: Zsolnay.

Hahnl, Hans Heinz (1985): Von der Diskreditierung der Ideologien. Erwartungshaltungen und literarische Produktion nach 1945. In: Waechter-Böhm, Liesbeth (hrsg.): Wien 1945. davor/ danach. Wien: Brandstätter, S. 153-163. 
Hahnl, Hans Heinz (1951): Zur Situation der Literatur. In: Stimmen der Gegenwart, S. 20-25.

Haut Commissariat de la République Française en Autriche; Division Information, Centre de Documentation (1948): Deux ans et demi de présence française en Autriche. Notes documentaires et études No 870; Série européenne - CXIV.

Heer, Friedrich (1985): Nach 1945. In: Jung, Jochen (hrsg.): Vom Reich zu Österreich. Kriegsende und Nachkriegszeit in Österreich erinnert von Augen- und Ohrenzeugen. München: dtv, S. 150-161.

Hussong, Marion (2000): Der Nationalsozialismus im österreichischen Roman 1945-1969. Tübingen: Stauffenburg. [= Stauffenburg-Colloquium; Bd. 52].

Klöckler, Jürgen (2004): Ici L’Autriche - Pays Ami! Frankreich und die Entnazifizierung in Österreich 1945/46. In: Schuster, Walter - Weber, Wolfgang (hrsg.): Entnazifizierung im regionalen Vergleich. Linz: Archiv der Stadt Linz, S. 455-472.

Kriegleder, Wynfrid (2014): Die Literatur der fünfziger Jahre in Österreich - ein Überblick. In: treibhaus 10 („Österreich“), S. 29-49.

Kunzelmann, Heide (2015): Make or Break? Instability as an Aesthetic Tool in Progressive Austrian Literature from 1945 to 1955. In: Journal of Austrian Studies 48, Nr. 2, S. 89-108.

Landa, Jutta (1988): Bürgerliches Schocktheater. Entwicklungen im österreichischen Drama der sechziger und siebziger Jahre. Frankfurt am Main: Athenäum. [= Literatur in der Geschichte. Geschichte in der Literatur 15].

Lebert, Hans (1960): Die Wolfshaut. Hamburg: Claassen.

Lernet-Holenia, Alexander (1945): Gruß des Dichters. In: Der Turm 1, Nr. 4/5, S. 109.

Lothar, Ernst (2018): Die Rückkehr; mit einem Nachwort von Doron Rabinovici. Wien: Zsolnay.

Lunzer, Heinz (1984): Der literarische Markt 1945-1955. In: Aspetsberger, Friedbert - Frei, Norbert - Lengauer, Hubert (hrsg.): Literatur der Nachkriegszeit und der fünfziger Jahre in Österreich. Wien: ÖBV. [= Schriften des Institutes für Österreichkunde 44/45], S. 24-45.

Maurer, Stefan - Neumann-Rieser, Doris - Stocker, Günther (hrsg.) (2017): Diskurse des Kalten Krieges. Eine andere österreichische Nachkriegsliteratur. Wien - Köln - Weimar: Böhlau. [= Literaturgeschichte in Studien und Quellen 29].

McVeigh, Joseph G. (1996): Lifting the Paper Curtain: The Opening of Austrian Literary Culture to Germany after 1945. In: German Studies Review 19, Nr. 3, S. 479-499.

McVeigh, Joseph (1988): „Das bin nur ich. Wenn ich es bin“: Politics and Literature in Austria after 1945. In: The German Quarterly 61, Nr. 1, S. 5-21.

McVeigh, Joseph (1988): Kontinuität und Vergangenheitsbewältigung in der österreichischen Literatur nach 1945. Wien: Braumüller. [= Untersuchungen zur österreichischen Literatur des 20. Jahrhunderts; Bd. 10].

Meissl, Sebastian - Mulley, Klaus-Dieter - Rathkolb, Oliver (hrsg.) (1986): Verdrängte Schuld, verfehlte Sühne. Entnazifizierung in Österreich 1945-1955 [= Symposion des Instituts für Wissenschaft und Kunst, Wien, März 1985]. Wien: Verlag für Geschichte und Politik.

Müller, Karl (1988): Die Bannung der Unordnung. Zur Kontinuität österreichischer Literatur seit den dreißiger Jahren. In: Stadler, Friedrich (hrsg.): Kontinuität und Bruch. 1938 - 1945 - 1955. Beiträge zur österreichischen Kultur- und Wissenschaftsgeschichte. Wien - München: Jugend und Volk, S. 181-215.

-n (1946): Zwei Bücher aus Österreich. Zur weltanschaulichen Situation unserer Zeit. In: Wiener Kurier, 28. 3. 1946.

o. V. (1946): Gerechtigkeit für Österreich! Rot-Weiss-Rot-Buch. Darstellungen, Dokumente und Nachweise zur Vorgeschichte und Geschichte der Okkupation Österreichs (nach amtlichen Quellen). Wien: Verlag der österreichischen Staatsdruckerei. 
Priester, Eva (1946): Die Aufgaben der österreichischen Literatur. In: Österreichisches Tagebuch, 16. 11. 1946.

Rabenstein-Michel, Ingeborg (2008): Bewältigungsinstrument Anti-Heimatliteratur. In: Germanica 42, S. 1-11. https://journals.openedition.org/germanica/525 (9. 1. 2019).

Rosenberg, Rainer (2003): Verhandlungen des Literaturbegriffs. Studien zu Geschichte und Theorie der Literaturwissenschaft. Berlin: Akademie Verlag.

[Sauter, Lilly von] L. v. S. (1948): Porträt unseres Helden. In: Europäische Rundschau 3, Nr. 17, S. 806-808.

Scharang, Michael (1986): Die proletarisierte Literatur. In: Scharang, Michael: Die List der Kunst. Essays. Darmstadt - Neuwied: Luchterhand, S. 14-24. [Zuerst in: Literatur Konkret 1983/1984, Nr. 8].

Schmid, Georg (1984): Die ,Falschen` Fuffziger. Kulturpolitische Tendenzen der fünfziger Jahre. In: Aspetsberger, Friedbert - Frei, Norbert - Lengauer, Hubert (hrsg.): Literatur der Nachkriegszeit und der fünfziger Jahre in Österreich. Wien: ÖBV. [= Schriften des Institutes für Österreichkunde 44/45], S. 7-23.

Schmidt-Dengler, Wendelin (1996): Bruchlinien. Vorlesungen zur österreichischen Literatur 1945 bis 1990 (2. Aufl.). Salzburg: Residenz.

Schmidt-Dengler, Wendelin (1995): Vorwort. In: Nachbaur, Petra - Scheichl, Sigurd Paul (hrsg.): Literatur über Literatur. Eine österreichische Anthologie. Innsbruck: Haymon, S. 11-24.

Schreiber, Hermann (1947): Das Buch. Papierpolitik. In: Österreichisches Tagebuch, 19. 9. 1947.

Stadler, Friedrich (1988): Kontinuität und/ oder Bruch? Anmerkungen zur österreichischen Wissenschaftsgeschichte 1938 bis 1955. In: Stadler, Friedrich (hrsg.): Kontinuität und Bruch. 1938 - 1945 - 1955. Beiträge zur österreichischen Kultur- und Wissenschaftsgeschichte. Wien München: Jugend und Volk, S. 9-23.

Stocker, Günther (2010): Der Kalte Krieg in der österreichischen Literatur. Ein Überblick. In: Hansel, Michael - Rohrwasser, Michael (hrsg.): Kalter Krieg in Österreich. Literatur - Kunst Kultur. Wien: Zsolnay. [= Profile 17/2010], S. 59-80.

[Torberg, Friedrich] tbg. (1954): Zur 30. Wiederkehr des Todestages von Franz Kafka. In: Forum 1, Nr. 6, S. 19.

United States Department of State (1963): Declaration on Austria. In: Franklin, William M. - Perkins, E. R. (hrsg.): Foreign Relations of the United States: Diplomatic Papers, 1943. General, Vol. 1. Washington: Government Printing Office, S. 761.

Weigel, Hans (1953): Die gefundene Generation: In: Kontinente 7, Nr. 1, S. 13-14.

Weigel, Hans (1953): Vorbemerkung. In: Stimmen der Gegenwart, S. 5-7.

Weigel, Hans (1951): Vorbemerkung. In: Stimmen der Gegenwart, S. 5.

Weigel, Hans (1950): Autoren, die uns nicht erreichen. Zur tragischen Situation der jungen österreichischen Literatur. In: Arbeiter-Zeitung, 24. 9. 1950.

Weigel, Hans (1946): Das verhängte Fenster. In: Plan 1, Nr. 5, S. 397-399.

Werner, Juliane (2021): Existentialismus in Österreich. Kultureller Transfer und literarische Resonanz. Berlin - Boston: De Gruyter. [= Studien und Texte zur Sozialgeschichte der Literatur; Bd. 153].

Wodianka, Stephanie (2018): Mythos und Tabula rasa: Poetik des Erzählens, Erinnerung und Gedächtnis, Fläche und Raum. In: Bizeul, Yves - Wodianka, Stephanie (hrsg.): Mythos und Tabula rasa. Narrationen und Denkformen der totalen Auslöschung und des absoluten Neuanfangs. Bielefeld: transcript, S. 25-39.

Zand, Herbert (1992): Letzte Ausfahrt. Roman der Eingekesselten. Wien et al.: Europaverlag.

Zeyringer, Klaus (2008): Österreichische Literatur seit 1945. Überblicke, Einschnitte, Wegmarken (ergänzte und aktualisierte Neuausgabe). Innsbruck: Studienverlag. 
Die Welt von Gestern und ihre Widersacher: (Dis)kontinuitäten in der österreichischen ...

Univ.-Ass. Dr. Juliane Werner / juliane.werner@univie.ac.at

Universität Wien, Abteilung für Vergleichende Literaturwissenschaft, Sensengasse 3a, 5.02, 1090 Wien, Österreich

This work can be used in accordance with the Creative Commons BY-SA 4.0 International license terms and conditions (https://creativecommons.org/licenses/by-sa/4.0/legalcode). This does not apply to works or elements (such as image or photographs) that are used in the work under a contractual license or exception or limitation to relevant rights 